$\underline{\text { Research article }}$

\title{
Comparison of a rapid immunochromatography assay with an enzyme linked immunosorbent assay (ELISA) for anti-dengue virus IgM detection
}

\author{
T Senaratne ${ }^{1}$, F Noordeen $^{1}$, N Dissanayake ${ }^{1}$, KGRA Kumara ${ }^{1}$ \\ Sri Lankan Journal of Infectious Diseases 2014 Vol.4 (2):77-82 \\ DOI: http://dx.doi.org/10.4038/sljid.v4i2.5925
}

\begin{abstract}
Objectives

The current study was undertaken to compare the sensitivity, specificity, positive predictive value and negative predictive values of a dengue IgM rapid immunochromatography (ICT) assay with a dengue $\operatorname{IgM}$ capture ELISA for the detection of anti-dengue virus IgM in patients clinically suspected of having dengue fever (DF).
\end{abstract}

\section{Methods}

Blood samples $(n=119)$ were collected from paediatric and adult patients suspected of having DF at Teaching Hospital, Peradeniya (THP) after 5 days of fever. The samples were analyzed for the presence of anti-dengue virus IgM using a commercial rapid dengue IgM ICT assay (Hexagon dengue, Human, Germany) and a commercial IgM capture ELISA (Panbio Diagnostics Inc, Australia) provided to THP by the Ministry of Health for the diagnosis of dengue fever.

\section{Results}

When tested using the rapid dengue IgM ICT assay, 80 blood samples were positive and 39 were negative for anti-dengue virus IgM. When tested using the dengue IgM ELISA, 100 sera were positive and 19 were negative for dengue IgM. Using the IgM capture ELISA as the comparator, the sensitivity and the specificity of the rapid assay were $79 \%$ and $94.7 \%$ respectively with a positive (PPV) and negative (NPV) predictive value of $98.8 \%$ and $46.2 \%$.

\section{Conclusion}

In comparison with the results of the IgM capture ELISA, the low sensitivity and NPV for antidengue virus IgM detection by the rapid dengue IgM ICT assay was noted. The low sensitivity and NPV means that patients with DF will be missed when using this test. In contrast, the high specificity and PPV indicate that this rapid assay is able to detect true positives. Since preliminary screening of DF is carried out widely using these rapid tests, the strengths and limitations of this and other similar tests require validation before use in diagnostic laboratories.

Keywords: Dengue fever, rapid tests, IgM ELISA, Sri Lanka

\begin{tabular}{l}
${ }^{1}$ Department of Microbiology, Faculty of Medicine, University of Peradeniya, Sri Lanka \\
\hline Address for correspondence: Dr. Faseeha Noordeen, Department of Microbiology, Faculty of Medicine, \\
University of Peradeniya, Sri Lanka. Telephone No: 94812396532 Email - faseeha.noordeen12@gmail.com
\end{tabular} 


\section{Introduction}

Dengue fever (DF) is a mosquito-borne viral infection transmitted through Aedes aegypti and Aedes albopictus. ${ }^{1}$ DF is caused by 4 distinct dengue virus (DENV) serotypes, namely DENV1, DENV2, DENV3 and DENV4. ${ }^{2}$

Clinical features of DENV infection may vary between mild self limiting DF, the more severe dengue hemorrhagic fever (DHF) and dengue shock syndrome (DSS). ${ }^{3}$ Epidemic DHF first started in Manila, Philippines from 1953 to 1954 and rapidly spread to Asian regions. ${ }^{4}$ DF is endemic in Sri Lanka and several outbreaks have been reported in the last ten years, with high morbidity and mortality rates recorded in the recent past. $^{5}$

In Sri Lanka, the laboratory diagnosis of DF is mainly by the detection of anti-dengue virus IgM antibodies using rapid immunochromatographic (ICT) assays. NS1 and dengue antibody detection are the 2 main methods used in the diagnosis of dengue infections. Although NS1 detection is available in private sector laboratories, it is not available in government hospitals for routine use. Most government hospitals also do not provide an antibody diagnostic service using ELISA based methods. However, rapid immunochromatographic (ICT) assays are being widely used in both government and private sector laboratories to provide a rapid diagnostic service.

There are many rapid IgM-based dengue diagnostic tests which are low cost, quick and easy to use. They exist in many lateral flow immunochromatographic strips formats. Most use recombinant antigens from all four dengue virus serotypes and provide results within 15 to 90 minutes. Evaluation of 4 such kits gave sensitivities ranging from 21\%-99\% and specificities from $77 \%-98 \%$ when compared with the gold standard ELISAs. ${ }^{6}$

There is no published data on the percentage of use of these methods for the detection of antidengue IgM antibodies in Sri Lanka. There is also a serious dearth of information on the reliability of these assays in Sri Lanka.

Thus the objective of the current study was to compare the sensitivity, specificity, positive predictive value (PPV) and negative predictive value (NPV) of one rapid dengue ICT IgM assay with a dengue IgM capture ELISA for the detection of anti-dengue virus IgM in clinically suspected DF patients. All the kits used for this study were provided to THP by the Ministry of Health, Sri Lanka.

\section{Materials and Methods}

Blood samples $(n=119)$ were collected between September 2011 and January 2012 from DF suspected paediatric and adult patients hospitalized at THP, Sri Lanka. The samples were analyzed for the presence of anti-dengue virus IgM and IgG using the rapid ICT IgM assay (Hexagon dengue, Human, Germany) and IgM capture ELISA (Panbio Diagnostics Inc, Australia). 
The rapid dengue ICT IgM and IgG assay was performed at the microbiology laboratory of THP as a part of the routine diagnosis of DENV infection, and the results of the patients were issued to the wards within 2-3 hours of the test.

The ELISA test was performed using the remaining serum at the Department of Microbiology, Faculty of Medicine, University of Peradeniya. Both assays were performed in accordance with the respective manufacturer's instructions.

\section{ICT rapid test for the detection of dengue IgG and IgM antibodies (Hexagon dengue, Human, Germany)}

This rapid assay can qualitatively detect IgG and IgM to DENV in human body fluids such as serum, plasma and whole blood. According to the manufacturer, antibodies to all 4 serotypes can be detected and no false positive results were obtained with yellow fever, Japanese encephalitis, Plasmodium falciparum and Plasmodium vivax. This rapid ICT assay captures dengue-specific IgM and IgG antibodies by the antigens coated on the colloidal gold particles. The immune complexes migrate along the membrane and are captured by monoclonal anti human IgG in the first line and monoclonal anti human IgM in the second line. The excess and the non-reacted particles will be captured by the anti dengue $\mathrm{IgG}$ forming the control line.

$10 \mu \mathrm{l}$ of patient serum was added to the sample window of the cassette immediately followed by the addition of 3-4 drops of diluent to the reagent window of the cassette. The test results were read at 15-20 minutes. If the sample contains both $\operatorname{IgG}$ and $\operatorname{IgM}, 3$ lines (IgG line, IgM line and control line) will be visible. If the sample contains only $\operatorname{IgG}$ or $\operatorname{IgM}$, only two lines will be visible (IgG or IgM line and control line) and if both antibodies are negative, only the control line will be visible.

\section{Dengue IgM capture ELISA (Panbio Diagnostics Inc, Australia)}

CDC recommends ELISA IgM detection for the detection of all 4 DENV serotypes. ${ }^{7}$ The Panbio Dengue IgM capture ELISA has been found to have high sensitivity and specificity. ${ }^{8}$ It was therefore chosen as the comparator test for the current study of a rapid immunochromatographic method widely used in Sri Lanka.

The test was carried out following the manufacturers' instructions. Briefly, an aliquot $(100 \mu \mathrm{l})$ of patient serum (diluted 1:100 in the diluent provided) was added to each well of the assay plate containing bound anti-human IgM which binds with human IgM in the patient's serum. The plate was incubated for 1 hour at $37^{\circ} \mathrm{C}$. The assay plate was washed with wash buffer and $100 \mu \mathrm{l}$ of previously mixed antigen-monoclonal antibody tracer solution was added.The plate was incubated for 1 hour at $37^{\circ} \mathrm{C}$ and washed again with wash buffer. The bound complexes were visualized by the addition of $100 \mu \mathrm{l}$ of tetramethyl-benzidine substrate per well. After 10 min, the reaction was stopped by the addition of $100 \mu \mathrm{l}$ of stop solution. The plates were read at 
$450 \mathrm{~nm}$ with a microtiter plate reader. The results were interpreted according to manufacturers' instructions. Positive and negative controls provided by the manufacturer were used.

Sensitivity, specificity, PPV and NPV were calculated for the ICT rapid test using the results generated by the IgM capture ELISA.

\section{Results}

Of the 119 samples received by the Microbiology Laboratory during the study period, 93 samples $(78.2 \%)$ were from the paediatric wards and only 26 samples $(21.9 \%)$ were from the

Table 1

Comparison of a rapid ICT assay with a standard ELISA for anti-dengue virus IgM detection

\begin{tabular}{ccc}
\hline & Rapid ICT assay & Rapid ICT assay \\
& $(+)$ & $(-)$ \\
ELISA (+) & 79 & 21 \\
ELISA (-) & 1 & 18 \\
\hline
\end{tabular}

adult wards. When tested by IgM ELISA, 100 samples were positive and 19 samples negative for dengue IgM.

Using the rapid dengue ICT IgM assay, 80 samples were positive and 39 were negative for antidengue virus IgM. (Table 1). Test accuracy indices were determined by calculating the sensitivity, specificity, positive predictive value (PPV), and negative predictive value (NPV) of the rapid dengue ICT IgM assay as shown in Table 2.

\section{Discussion}

Table 2

DF is considered as one of the most important vector borne infectious diseases in Sri Lanka. High morbidity and mortality caused by DF in the past has declined in recent times, possibly due to adherence to new management guidelines.

The ELISAs have been widely used in many DF endemic countries for anti-dengue virus $\mathrm{IgM} / \mathrm{IgG}$ detection. The use of ELISA has the advantage of testing a large sample size at the same time. ${ }^{9}$ However; this method requires more expensive laboratory infrastructure and expertise. Considering these factors, ELISA is considered more suitable for use in a tertiary care hospital laboratory which receives a large number of samples for routine laboratory diagnosis of DENV infections.

To meet the gap in diagnostics, in many dengue endemic developing countries, detection of DENV infection in the laboratory is carried out using the rapid ICT assays. The rapid dengue assay is designed to detect both IgM and IgG simultaneously. Based on this, the use of rapid ICT assays reduces the overall cost and the time for testing two immune markers in DF. Some manufacturers claim that these kits are also able to differentiate primary DENV infections from 
secondary DENV virus infections. ${ }^{10}$ Nevertheless, findings of $\mathrm{Nga}$ et $\mathrm{al}^{11}$ show that the discrimination of acute primary and secondary DENV infection in DF is unreliable when using the rapid ICT assays.

The availability of many rapid ICT assays in the market suggests the need for validating these assays by independent bodies to determine their performance and ensure reliability of the in-use assays. In addition, these ICT assays should be regulated nationally to prevent the introduction of unregulated assays to the market. In the absence of a national regulatory system, evaluation by researchers in different locations of the country would be useful. ${ }^{8}$ Validating widely used rapid assays for anti-dengue virus IgM detection would increase the reliability of the results and help support the clinical diagnosis. Validating rapid ICT assays are usually done by comparing the accuracy indices such as sensitivity, specificity, PPV and NPV with a known reference standard or a gold standard method. ${ }^{6}$ Using a known reference standard method might be difficult unless routinely performed by a national laboratory for quality control of assays used in a country. Thus a centrally validated (CDC) and widely accepted ELISA method is helpful for use as a comparator to assess rapid ICT assays. ${ }^{12,13}$

Some studies have shown high rates of sensitivity and specificity for rapid ICT assays.9 However, in the current study, the sensitivity and the NPV for anti-dengue virus IgM detection by the rapid ICT assay were comparatively lower than that of ELISA (Table 2) with the inference that this particular assay will give a high percentage of false negatives.

Only one rapid ICT (Hexagon dengue, Human, Germany) was tested in this study. However, there are many ICT cassettes (at least five different brands) marketed and utilized in the Sri Lankan market. There is no quality assurance programme in Sri Lanka currently for the testing and validation of imported diagnostic kits. The results of this study suggest the need for such a programme to be established so that users, including laboratories, clinicians and patients would be aware of the limitations of the test being used for diagnosis of a commonly encountered infection in Sri Lanka.

The limitations of the current study include sample size and the testing of only one rapid ICT kit.

\section{Conflict of interest}

None declared by the authors.

\section{Ethics}

The rapid ICT assay was performed as part of the routine diagnosis of dengue virus infection and the rest of the serum was used for the ELISA. Hence ethical approval was not requested for this study. 


\section{References:}

1. Guzman MG, Halstead SB, Artsob H, et al. Dengue: a continuing global threat. Nature Reviews Microbiology. 2010; 8(12):S7-S16. doi:10.1038/nrmicro2460

2. Chau TNB, Quyen NTH, Thuy TT, et al. Dengue in Vietnamese infants--results of infectionenhancement assays correlate with age-related disease epidemiology, and cellular immune responses correlate with disease severity. The Journal of infectious diseases. 2008; 198(4):516-24. doi: http://dx.doi.org/10.1086/590117

3. Martina BEE, Koraka P, Osterhaus ADME. Dengue virus pathogenesis: an integrated view. Clinical Microbiology Reviews.2009; 22(4):564-581. doi: http://dx.doi.org/10.1128/CMR.00035-09

4. Gubler DJ. Dengue and dengue hemorrhagic fever. Clinical Microbiology Reviews. 1998; 11(3):480-96. No doi

5. Kularatne SAM, Seneviratne SL, Malavige GN, et al. Synopsis of findings from recent studies on dengue in Sri Lanka. Dengue bulletin 2006; 30:80-86. No doi

6. Peeling RW, Artsob H, Pelegrino JL,et al. Evaluation of diagnostic tests: dengue. Nature Reviews Microbiology, 2010; S30-S37. doi:10.1038/nrmicro2459

7. Laboratory guidance and diagnostic testing, CDC Atlanta. Available at http://www.cdc.gov/dengue/clinicalLab/laboratory.html

8. Sang CT, Cuzzubbo AJ, and Devine PL. Evaluation of a commercial Capture EnzymeLinked Immunosorbent assay for detection of Immunoglobulin $\mathrm{M}$ and $\mathrm{G}$ antibodies produced during dengue infection. Clin Diagn Lab Immunol. 1998; 5(1): 7-10. No doi

9. Sekaran SD, Lan EC, Subramaniam G. Comparison of five serological diagnostic assays for the detection of $\operatorname{IgM}$ and $\mathrm{IgG}$ antibodies to dengue virus. African Journal of Microbiology Research 2008; 2:141-147 No doi

10. Blacksell SD. Commercial dengue rapid diagnostic tests for point-of-care application: Recent evaluations and future needs? Journal of Biomedicine and Biotechnology. 2012; Article ID $151967: 12$ pages doi: http://dx.doi.org/10.1155/2012/151967

11. Nga TTT, Thai KTD, et al. Evaluation of two rapid immunochromatographic assays for diagnosis of dengue among Vietnamese febrile patients. Clinical and Vaccine Immunology 2007; 14(6):799-801. doi :10.1128/CVI.00483-06

12. Andries A, Duong V, Ngan C, et al. Field evaluation and impact on clinical management of a rapid diagnostic kit that detects dengue NS1, IgM and IgG. PLOS Neglected Tropical Diseases 2012; 6(12):1-9 doi: 10.1371/journal.pntd.0001993

13. Pan-ngum W, Blacksell SD, Lubell Y, et al. Estimating the true accuracy of diagnostic tests for dengue infection using Bayesian Latent Class Models. $\quad$ Plos One 2013; 8(1):1-7. doi: 10.1371/journal.pone.0050765 